\title{
Investigation of Optical Interconnection by using Photonic Wire Bonding
}

\author{
ZhiChen $\mathrm{GU}^{1}$, Tomohiro AMEMIYA ${ }^{2}$, Atsushi ISHIKAWA ${ }^{3}$, Yuki ATSUMI ${ }^{1}$, JoonHyun $\mathrm{KANG}^{1}$, Takuo HIRATANI ${ }^{1}$, \\ Yusuke HAYASHI $^{1}$, Junichi SUZUKI ${ }^{1}$, Nobuhiko NISHIYAMA ${ }^{1}$, Takuo TANAKA ${ }^{3}$, and Shigehisa ARAI ${ }^{1,2}$ \\ 1-Department of Electrical and Electronic Engineering, Tokyo Institute of Technology, Tokyo 152-1552, Japan \\ 2-Quantum Nanoelectronics Research Center, Tokyo Institute of Technology, Tokyo 152-1552, Japan \\ 3-Metamaterials Laboratory, RIKEN, Saitama 351-0198, Japan \\ Main author email address: gu.z.ab@m.titech.ac.jp
}

\begin{abstract}
Photonic wire bonding based on direct laser writing of three-dimensional polymer structure was discussed as one of practical approaches for photonic integration of heterogeneous optical components. Here we assessed the coupling efficiency between photonic wire bond and III-V/Si optical components, and demonstrate the fabrication of photonic wire bond between two III-V semiconductor chips.
\end{abstract}

DOI: 10.2961/jlmn.2015.02.0007

Keywords: Photonic integration, Photonic wire bonding, direct laser writing, III-V semiconductor, Si photonics

\section{Introduction}

As data rates in information processing systems continue to grow exponentially, much effort has been devoted to photonic integration because the fundamental limitations of electrical interconnects (e.g. bandwidth, spatial density, and power consumption) become obvious. Starting from the research of monolithic integration of III$\mathrm{V}$ compound components on InP-base platform, great progress has been made during the past few decades [1-4]. Currently the monolithic integration based on silicon photonics is considered to be the most promising option which enables high density photonic-electronic integration by utilizing mature CMOS technology [5-8]. However, photonic integration of heterogeneous optical components (III-V/Si) is still challenging. Previous research activities in this field primarily concentrated on the hybrid integration of $\mathrm{Si}$ and III-V components which were separately fabricated by electron-beam (EB) lithography and organometallic vapor phase epitaxy (OMVPE), respectively. Recently two primary techniques as known as direct bonding $[9,10]$ and polymer bonding $[11,12]$ have been vigorously studied in order to realize hybrid integration of III-V/Si optical components. However, either of them have structure restriction, which tend to be the barriers to effective use, to achieve good optical coupling.

In this paper, we present an approach by utilizing direct laser writing of three-dimensional polymer structures by using two photon absorption. Photonic wire bonding (PWB), which is a 3D-freeform transparent polymer waveguide as the counterpart of metal wires in electronic devices, has been proposed to connect optical components located on different chips [13-15]. Direct 3D writing with intense femtosecond laser pulses has been used to create the structure of the PWB. Because of the nonlinear multiphoton-absorption process, the modification only occurs at the focusing voxel of the laser beam, and we can form submicrometer-scaled polymer waveguides which would be able to allow single-mode propagation in it regarding low-loss integration. Here, by choosing SU-8 ( $n$ $=1.57$ ) as the polymer material, we consider the possibility of PWB for realizing 3D-freeform and low-loss hybrid photonic integration from the theoretical analysis and

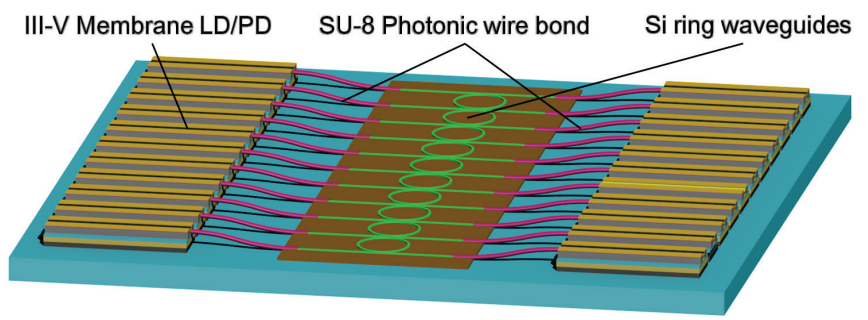

Fig. 1 Concept of photonic integrated circuit (PIC) based on PWB.

finally prove the feasibility of this method by connecting several III-V-based on-chip optical devices.

\section{Research concept}

Fig. 1 indicates a concept of the heterogeneous photonic integration based on PWB. On-chip active optical devices such as III-V compound light sources/detectors as well as the passive optical devices such as Si waveguides or arrayed waveguide grating would be necessary to realize a photonic integrated circuit (PIC), the optical signal is expected to be emitted from a laser, passing through lowloss passive components on Si-photonics platform and finally detected by a high-sensitivity photo-detector. These on-chip devices are designed to be mounted on the common submount by Benzocyclobutene (BCB) adhesive bonding and then embedded into the photosensitive resist SU-8. Direct laser writing by intense femtosecond laser beam is then used to build 3D-freeform PWB in the volume of the resist to connect separated optical components located on different chips [16-21].

However, the coupling loss between such polymer waveguides and optical devices would be important to realize the high-performance PICs. In the next sections, we show the theoretical analysis of coupling loss and the feasibility of low-loss heterogeneous photonic integration based on PWB. 


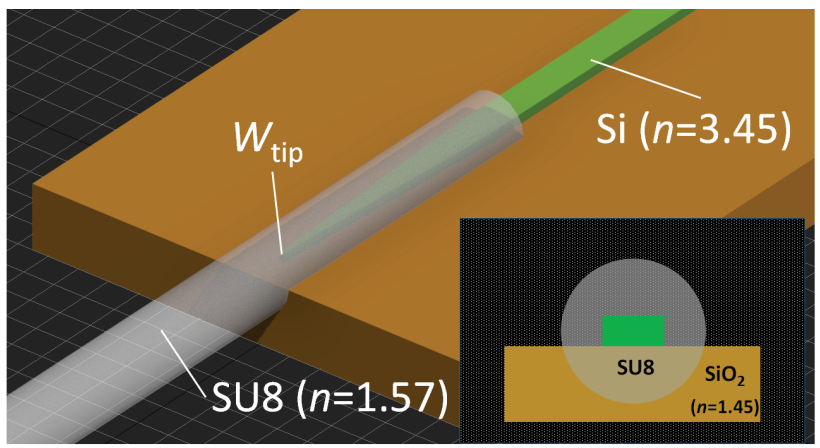

Fig.2 Numerical simulation model of the transition section between a $\mathrm{Si}$ waveguide and a polymer PWB waveguide.

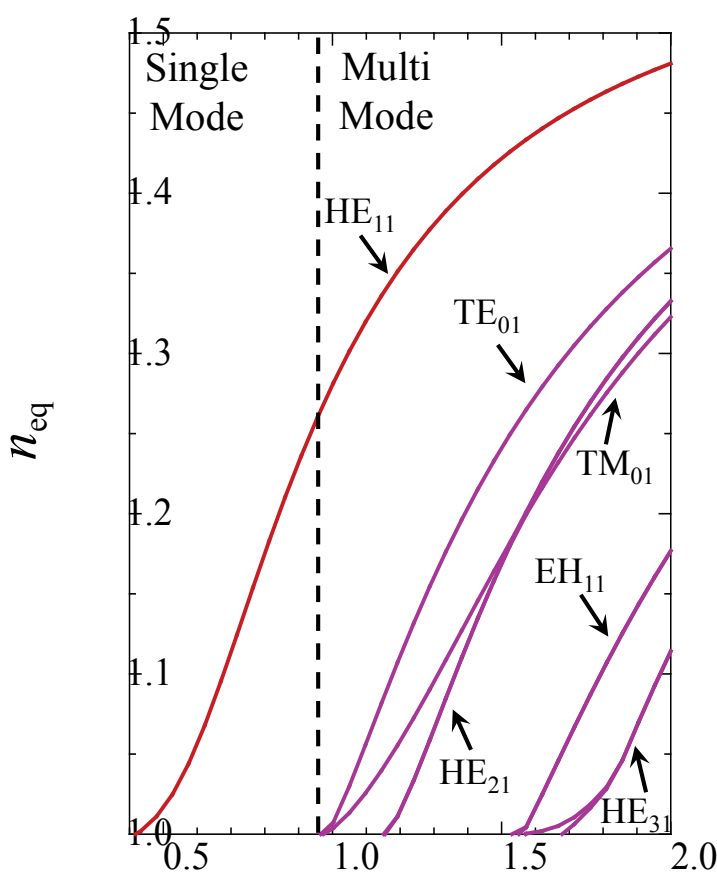

Diameter of Photonic wire bond $(\mu \mathrm{m})$

Fig.3 The calculated equivalent refractive index $n_{\text {eq }}$ as a function of the diameter of PWB for each mode.

\section{Modeling and design}

In this section, we discuss the coupling structure between PWB and III-V/Si optical components based on calculation results in order to obtain minimum coupling loss.

\subsection{Coupling loss between PWB and Si waveguides}

Fig. 2 indicates the simulation model of the coupling structure between PWB and $\mathrm{Si}$ waveguides. Here, we introduced a $\mathrm{Si}$ waveguide tip in this structure in order to reduce the reflection loss caused by large refractive-index difference between $\mathrm{Si}(n=3.45)$ and SU-8 $(n=1.57)$. Also, an excess loss caused by mode conversion can be avoided by single-mode propagation in both $\mathrm{PWB}$ and $\mathrm{Si}$ waveguides. The Si waveguide is typically $500 \mathrm{~nm}$ wide and $220 \mathrm{~nm}$ high which satisfy the condition of singlemode propagation, and tapered down over a length of 15 $\mu \mathrm{m}$ to an optimized tip width $W_{\text {tip. }}$. For single-mode PWB, a certain kind of small waveguide cross section has to be

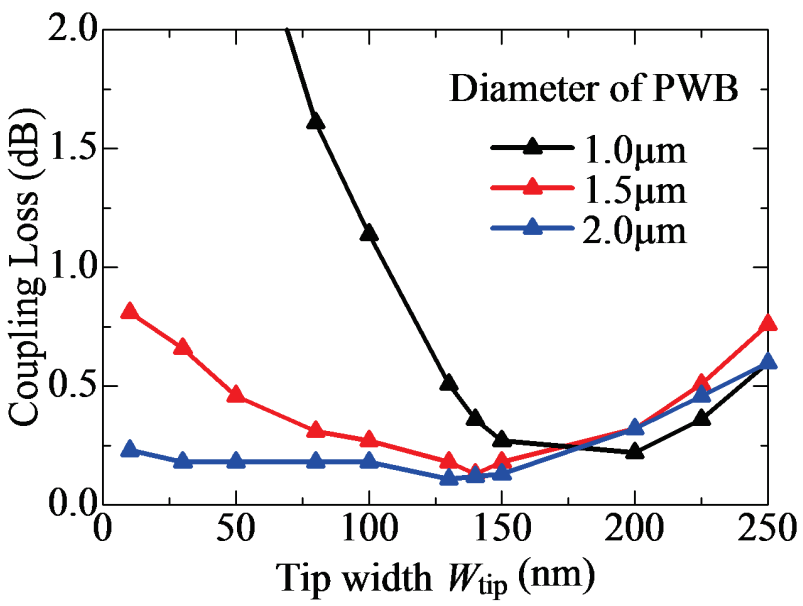

Fig.4 Tip width $W_{\text {tip }}$ dependency of the coupling loss calculated by FDTD method with different diameters of PWB near the condition of single-mode propagation.

chosen. Here we assumed a circular cross section similar to the optical fiber and used a commercially available finite difference method (FDM) solver (FIMMWAVE by Photon Design) to figure out the mode characteristic as a function of the diameter of the PWB at the wavelength of $1.55 \mu \mathrm{m}$. Fig. 3 shows the calculated equivalent refractive index $n_{\mathrm{eq}}$ as a function of the diameter of PWB for each mode, which indicates that multi-mode propagation could be suppressed when the diameter of the PWB is around $1 \mu \mathrm{m}$.

As one of crucial parameters in this transition section, the tip width $W_{\text {tip }}$ was varied between $10 \mathrm{~nm}$ and $250 \mathrm{~nm}$ and the optimized value was determined by another simulator based on finite difference time domain (FDTD) method (FullWAVE by RSoft). According to the calculation result represented in Fig. 4, the coupling loss below $0.25 \mathrm{~dB}$ can be obtained when the tip width $W_{\text {tip }}$ is $130 \mathrm{~nm}$ and embedded in a $1.5 \mu \mathrm{m}$-diameter or $2.0 \mu \mathrm{m}$-diameter PWB. Although the PWB smaller than $1-\mu \mathrm{m}$-diameter clearly suppresses the excitation of higher order modes, extremely small cross section would also weaken the optical confinement which leads to an increase of the coupling loss. On the other hand, it seems like that the PWB with larger diameter would provide better coupling efficiency. However the coupling to higher-order modes was included in this calculation result, which would cause an increase of the coupling loss if only the coupling to the fundamental mode is considered. The calculation result based on the FDM solver told us that $95 \%$ of the coupled light would contribute to the fundamental mode transmission of 1.5$\mu \mathrm{m}$-diameter PWB and $90 \%$ in the case of $2.0-\mu \mathrm{m}$-diameter

Next, we calculated the tolerance of the coupling loss considering the displacement between PWB and $\mathrm{Si}$ waveguides because the alignment accuracy of the endpoint of PWB waveguide before direct laser writing is completely determined by the inverted microscope. It is considered that by applying an objective lens of $\times 40$ magnification to the microscope, the displacement in all three dimensions could be suppressed within an acceptable range of less than $\pm 500 \mathrm{~nm}$. The calculation results of the relationship between the coupling loss and the displacement in vertical and horizontal directions are shown in Figs. 5(a) and 5(b), respectively. The bilateral 

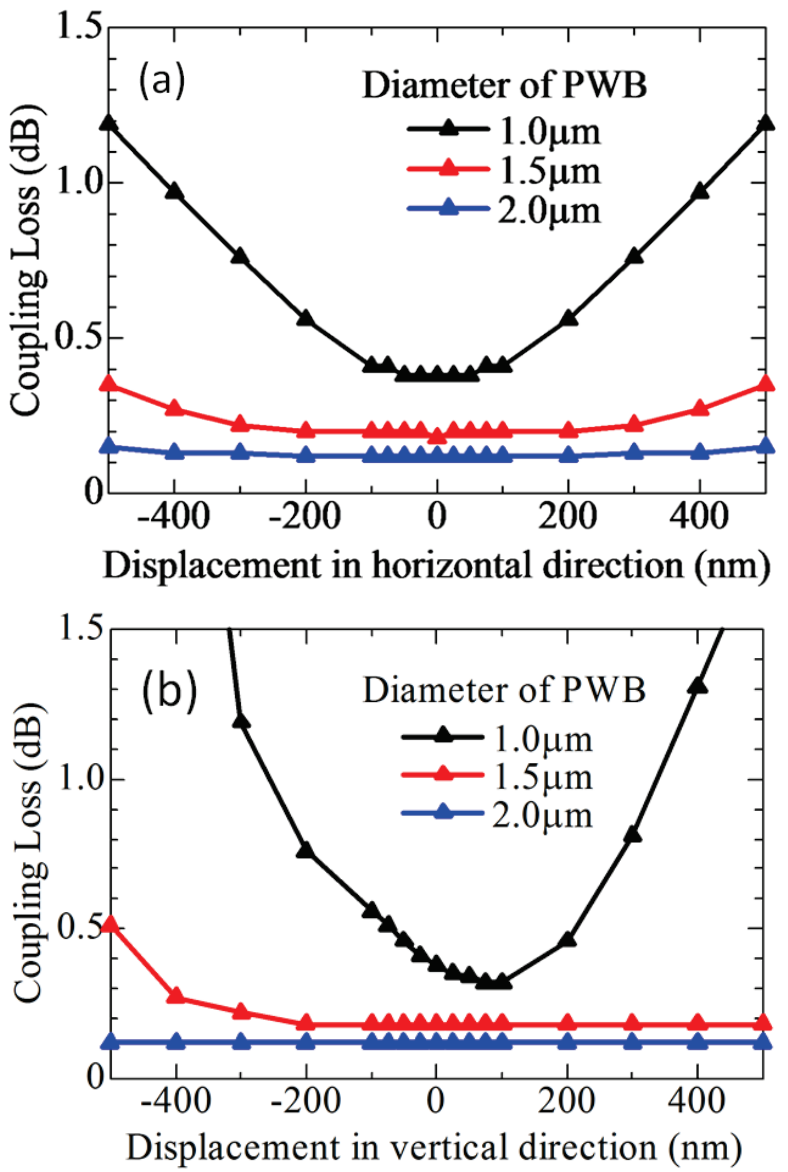

Fig.5 Coupling loss related to the displacement of PWB and $\mathrm{Si}$ waveguide in both (a) horizontal and (b) vertical directions calculated by FDTD method with different diameters of PWB near the condition of single mode propagation.

symmetric coupling structure as shown in Fig. 2 indicates that the variation of displacement in both positive and negative horizontal direction would have the same effect on the coupling loss as the anticipated result shown in Fig. 5(a). On the other hand, due to the asymmetric refractiveindex distribution of the $\mathrm{Si}$ waveguide ( $\mathrm{Si}: n=3.45$ on $\mathrm{SiO}_{2}: n=1.45$ ) in the vertical direction, the calculation result in Fig. 5(b) shows that the displacement in negative vertical direction would cause worse degradation of the coupling efficiency compared to the positive direction. Unevenness tolerance characteristics in both figures are merely caused by the extremely small cross section when the diameter of PWB is $1 \mu \mathrm{m}$. However, it is indicated that complanate and favorable characteristics could be obtained if the diameter of the PWB is larger than $1.5 \mu \mathrm{m}$. In consideration of all the facts above, we choose the diameter of the PWB to be $1.5 \mu \mathrm{m}$. Under this condition, the coupling loss remains below $0.5 \mathrm{~dB}$ against the variation of the displacement in both vertical and horizontal directions.

\subsection{Coupling loss between PWB and III-V lateral current injection membrane laser}

Based on the numerical analyses mentioned above, here we fix the diameter of the PWB to be $1.5 \mu \mathrm{m}$, which would also be used in the assessment of the coupling loss between III-V compound laser and a PWB polymer waveguide. The

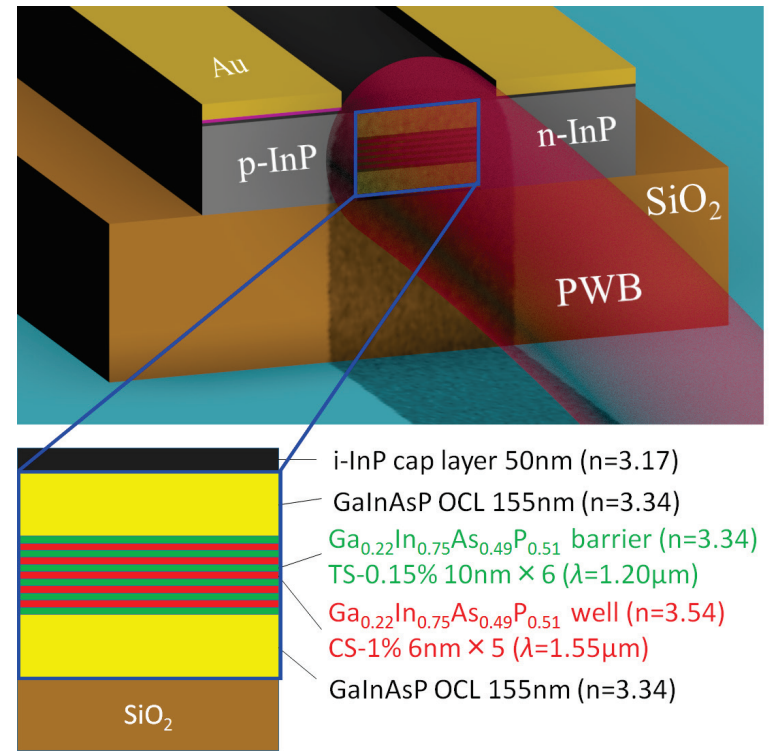

Fig.6 Simulation model of the transition section between a Lateral current injection (LCI) FP membrane laser and a polymer PWB waveguide.
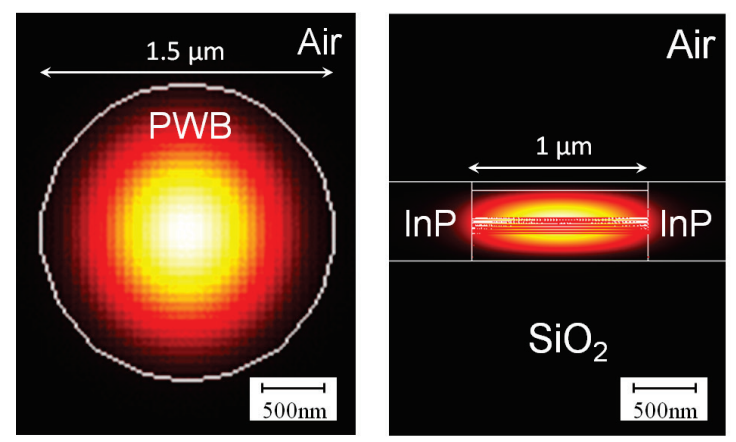

Fig.7 Mode distribution in the core layer of LCI membrane laser and PWB.

designed coupling structure of a lateral current injection (LCI) membrane FP laser and a PWB is shown in Fig. 6, where the membrane laser consists of a thin semiconductor core layer sandwiched by polymer claddings such as air, $\mathrm{BCB}$, or $\mathrm{SiO}_{2}$. Therefore, an optical confinement factor to the active region of the membrane laser is enhanced to approximately 3 times higher than that of conventional double-heterostrucure lasers because of the high-index contrast between the core and claddings [22]. Moreover, the active region volume and mirror loss can be reduced by adopting a DFB laser cavity with wirelike active regions [23]. A lower threshold current operation is expected with these effects, leading to ultralow power consumption of less than $100 \mathrm{fJ} /$ bit and fulfill the demand of the light source for PICs. Due to the insulation property of the cladding layers in this structure, a LCI structure should be introduced [24-26] in order to achieve an electricalinjection-type membrane laser which enables the current flow via $p-n$ junctions on either side of the core layer. Here, we assume the GaInAsP/InP LCI FP membrane laser in this simulation model which is the same as that of our previous device $[27,28]$. 


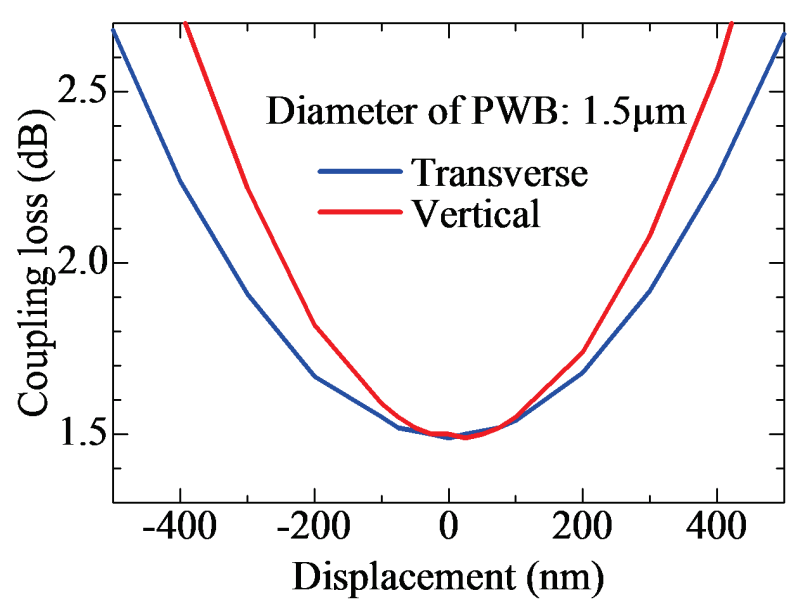

Fig.8 Coupling loss related to the displacement of the center of PWB and LCI Membrane laser in both vertical and transverse direction calculated by overlap integral.

The cross-sectional structure of the core layer in this LCI-FP membrane laser is also illustrated in Fig. 6. The core layer consists of five 1\% compressively-strained (CS) $\mathrm{Ga}_{0.22} \mathrm{In}_{0.78} \mathrm{As}_{0.81} \mathrm{P}_{0.19}$ quantum-wells (CS-5QWs, 6-nmthick, $\left.\lambda_{\mathrm{g}}=1220 \mathrm{~nm}\right), \quad 0.15 \%$ tensile-strained (TS) $\mathrm{Ga}_{0.26} \mathrm{In}_{0.74} \mathrm{As}_{0.49} \mathrm{P}_{0.51}$ barriers (10-nm-thick, $\left.\lambda_{\mathrm{g}}=1550 \mathrm{~nm}\right)$, sandwiched by optical confinement layers (OCLs, 155${ }^{\mathrm{i}} \mathrm{nm}$-thick for both sides). The total thickness of the core layer is $450 \mathrm{~nm}$ including a 50 -nm-thick InP cap layer for surface passivation. Top and bottom cladding layers were composed of air $(n=1)$ and $\mathrm{SiO}_{2}(n=1.45)$, respectively. We assumed the stripe width of the active region to be 1.0 $\mu \mathrm{m}$ and calculated the mode distribution in the core layer by using FDM solver. The calculation result is illustrated in Fig. 7.

In terms of Fabry-Perot laser, the induced light is directly emitted from the facet of the active region. Thus, we considered an end face contact between the PWB and the core layer of the LCI-FP membrane laser for higher coupling efficiency. Here, we ignored the influence of higher-order modes and calculated the coupling efficiency of this structure by integral of the mode distribution in the core layer of the LCI-FP membrane laser and the PWB over the interface. The tolerance characteristic can also be solved out in the same way by adjusting the relative position of the center of the PWB and the core layer. The calculation result is shown in Fig. 8. The coupling structure is also bilateral symmetrical as same as the one with $\mathrm{Si}$ waveguide, which has the same effect on the coupling loss in both positive and negative horizontal directions. The minimum coupling loss of $1.5 \mathrm{~dB}$ can be obtained when there is no displacement. Compare to the discussion in section 3.1, the reason why the coupling efficiency between Si waveguide and PWB is much higher than the case of III$\mathrm{V}$ membrane laser is that we used spot size converter to efficiently couple light from the strongly confined high index contrast silicon waveguide into the PWB, which has a much larger mode field size. On the other hand, the end face contact between the PWB and the core layer of the membrane laser would have no contribution to the enhancement of coupling efficiency.

However, the original performance of FP laser itself can't be maintained after bonding because one of the facet

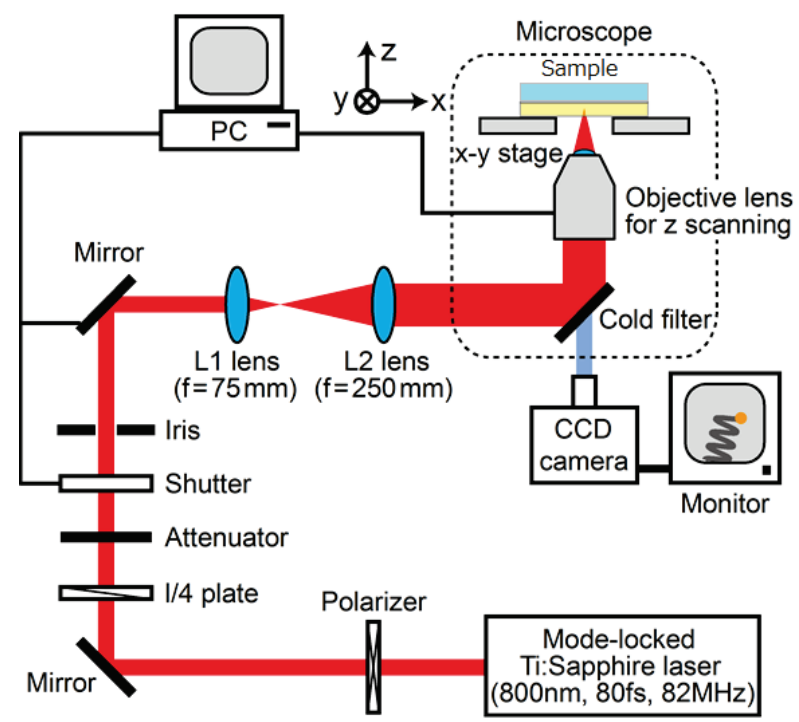

Fig.9 Optical system used for direct 3D laser writing of photonic wire bond.

Table 1 PWB geometry based on different fabrication conditions.

\begin{tabular}{ccccc}
\hline & $P=110 \mathrm{~mW}$ & $P=88 \mathrm{~mW}$ & $P=70 \mathrm{~mW}$ & $P=55 \mathrm{~mW}$ \\
\hline NA & $2.7 \mu \mathrm{m}$ & $1.8 \mu \mathrm{m}$ & Fail & Fail \\
0.50 & & & & \\
NA & $2.4 \mu \mathrm{m}$ & $2 \mu \mathrm{m}$ & Fail & Fail \\
0.95 & & & &
\end{tabular}
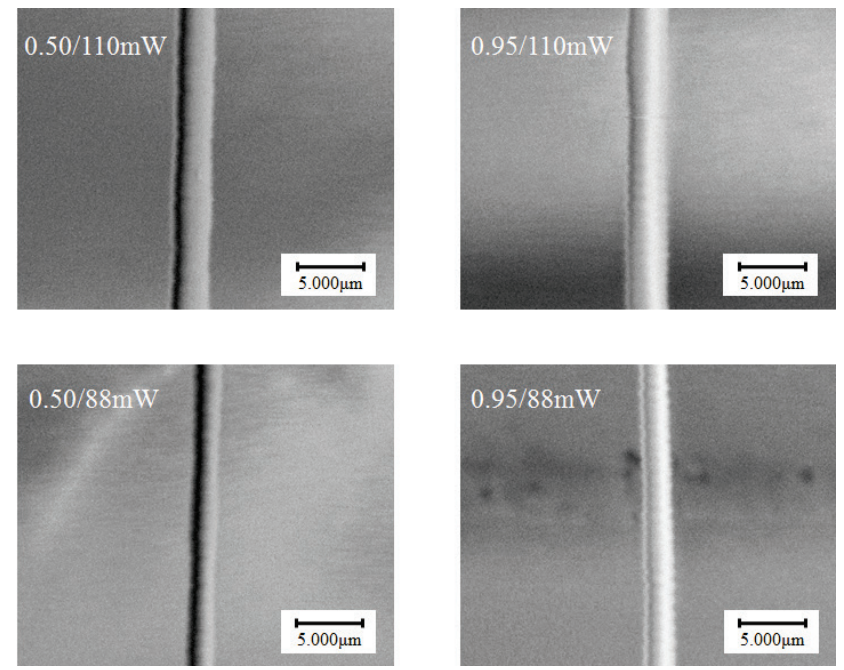

mirrors is exchanged with the polymer material SU-8 instead of air which leads to the variation of surface reflection ratio. In order to overcome this obstacle, we are now considering a new coupling structure based on the LCI DFB structure with butt-joint III-V waveguide.

\section{Experimental result}

Fig. 9 shows the optical direct laser writing system consisting of a mode-locked Ti: sapphire laser with the lasing wavelength of $800 \mathrm{~nm}$, the pulse width of $80 \mathrm{fs}$, and the repetition rate of $82 \mathrm{MHz}$. The laser beam was focused in the volume of SU- 8 and induced two-photon absorption by using objective lens inverted microscope which can also 

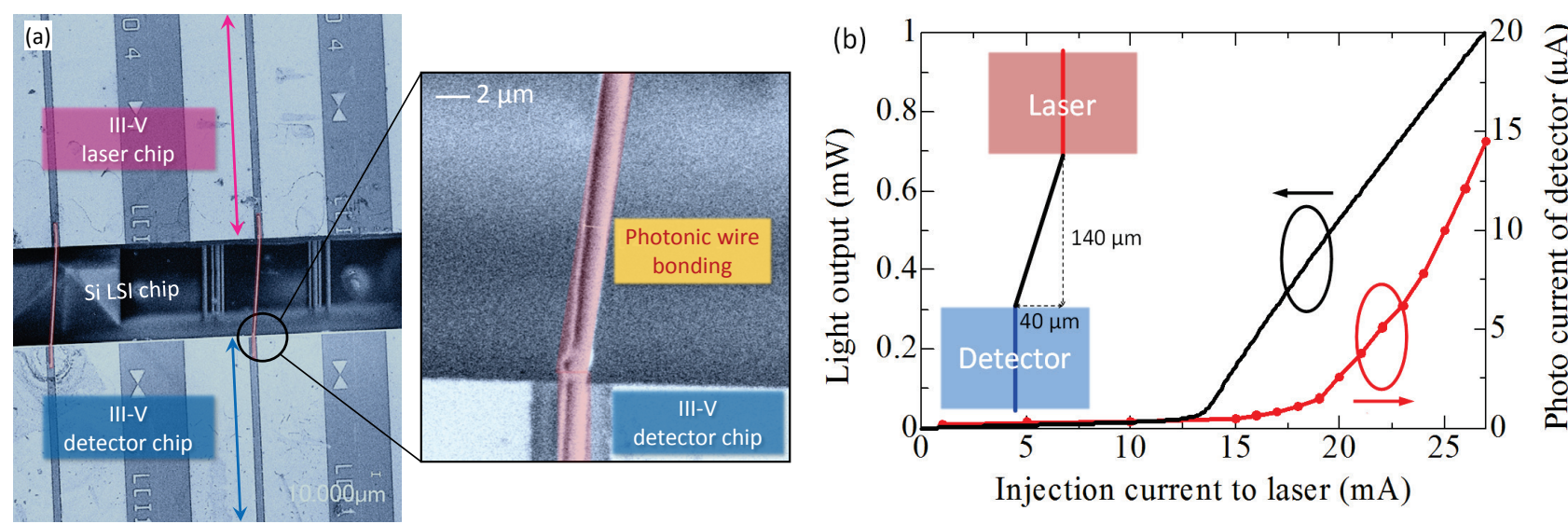

Fig. 10 (a) Two III-V optical chips were fixed on LSI host substrate by BCB adhesive bonding and connected by a photonic wire bond with $2 \mu \mathrm{m}$ diameter. (b) Transmission characteristic between two III-V optical chips through PWB.

be used to observe the actual positions of the coupling structures. The sample was supported by a piezo x-y stage and scanned in three dimensions. The PWB polymer waveguide geometry can be adapted to the observed relative positions of the two optical components by configuring the scan path of laser beam through computer control. Therefore, high-precision alignment of optical devices when settling them on the host substrate would be obsolete, which paves the way to 3D-freeform photonic integration of heterogeneous optical devices.

For a proof-of-principle experiment, in order to figure out the optimized condition of direct laser writing which can form the closest shape of simulation, we firstly fabricated PWB waveguides between two dummy chips with different laser powers $P$ by using objective lens with different numerical apertures (NA) of 0.95 and 0.5 . The scanning speed of the laser beam was set to be $10 \mu \mathrm{m} / \mathrm{s}$ and the interval between two chips was $80 \mu \mathrm{m}$. Variation of the PWB width based on different fabrication condition is listed in Table 1 together with their scanning electron microscope (SEM) images captured from top view. The width of the PWB ranges from $1.8 \mu \mathrm{m}$ to $2.7 \mu \mathrm{m}$ during the variation of fabrication condition. We couldn't discern PWBs fabricated with the laser power below $88 \mathrm{~mW}$. In order to obtain the ideal cylindrical PWB as with design, we considered to introduce an objective lens with higher NA in the fabrication which supposed to provide lower aspect ratio of the cross section of PWB [29]. For now, we determined the laser power to be $88 \mathrm{~mW}$ and NA=0.95 as an appropriate condition of direct laser writing, which provide us the closest shape of PWB after fabrication compared to the design. The width of PWB under this fabrication condition would be $2 \mu \mathrm{m}$ as shown in Table 1 and indeed couldn't satisfy the condition of ideal crosssectional shape of PWB according to the discussion in section 3.1. In the future work, we consider the possibility of realizing the 1.5- $\mu \mathrm{m}$-diameter PWB as designed by controlling the exposure dose with the writing speed.

Based on the condition setting mentioned above, we actually fabricated PWB between two LCI FP laser and detector chips (the same structure with laser chips) as shown in Fig. 10(a). The height of chips was exactly the same and the interval between two chips was $140 \mu \mathrm{m}$. In order to figure out the feasibility of PWB regarding optical transmission between laser and detector chips, we intensively shift the relative position of their core layer in the transverse direction for $40 \mu \mathrm{m}$ when we located them on the Si host substrate by BCB adhesive bonding. Fig. 10 (b) shows the photocurrent of the detector regarding to the current injected into the LCI FP laser (The I-L characteristic of this laser is also provided in the figure for comparison). For reference, the measured photocurrent stayed below measurement limitation $(0.2 \mu \mathrm{A})$ in another laser-detector set without PWB between them. According to this figure, we finally confirmed the optical transmission between these two optical components through PWB.

\section{Summary}

In this paper, we discussed photonic wire bonding as a practical approach to realize heterogeneous photonic integration. Direct laser writing based on two photon absorption was introduced to fabricate three-dimensional freeform PWBs. The coupling loss lower than $-0.5 \mathrm{~dB}$ between single-mode $\mathrm{PWB}$ and $\mathrm{Si}$ waveguide, $-1.5 \mathrm{~dB}$ between PWB and III-V LCI-FP membrane laser was obtained by simulation, respectively. In the experiment, we fabricated a PWB with $2 \mu \mathrm{m}$ width and successfully connected two III-V semiconductor optical chips fixed on LSI host substrate by BCB adhesive bonding. And we confirmed the optical transmission between those two LCI FP laser and detector chips through PWB.

While this work demonstrates the basic viability of PWB for heterogeneous photonic integration, realization of completely single mode propagation in photonic wire bond and the coupling structure which can maintain the original performance of III-V compound devices is still worth studying. Moreover, high speed optical transmission through photonic wire bond is essential in order to obtain high performance photonic integrated circuits, further investigations are necessary to systematically assess the transmission characteristic of PWB.

\section{Acknowledgments}

This work was supported by JSPS KAKENHI Grant Numbers \#24246061, \#25709026, \#25420321, \#13J08096, \#14J02327.

\section{References}

[1] A. Umbach, S. Van Waasen, U. Auer, H.-G. Bach, R. M. Bertenburg, V. Breuer, W. Ebert, G. Janssen, G. G. 
Mekonnen, W. Passenberg, W. Schlaak, C. Schramm, A. Seeger, F. J. Tegude, and G. Unterborsch: Electron. Lett., 32, (1996) 2142.

[2] K. J. Chen, K. Maezawa, and M. Yamamoto: IEEE Electron Dev. Lett., 17, (1996) 127.

[3] R. Gibis, H. Kizuki, P. Albrecht, P. Harde, G. Urmann, R. Kaiser, and H. Künzel: J. Cryst. Growth, 209, (2000) 463.

[4] M. Hamacher, R. Kaiser, H. Heidrich, P. Albrecht, B. Borchert, K. Janiak, R. Loffler, S. Malchow, W. Rehbein, and H. Schroeter-Janssen: Proc. 12th International Conference on Indium Phosphide and Related Materials, (2000) 21.

[5] R. A. Soref: IEEE J. Sel. Top. Quantum Electron., 12, (2006) 1678

[6] C. Gunn: IEEE Micro, 26, (2006) 58.

[7] R. M. Kubacki: Proc. 56th Electronic Components and Technology Conference, (2006) 776.

[8] B. Jalali, T. Indukuri, P. Koonath, and K. Kishima: Proc. SPIE, 6183, (2006) 618314.

[9] A. W. Fang, H. Park, O. Cohen, R. Jones, M. J. Paniccia, and J. E. Bowers: Opt. Express, 14, (2006) 9203.

[10] T. Okumura, T. Maruyama, H. Yonezawa, N. Nishiyama, and S. Arai: IEEE Photon. Technol. Lett., 21, (2009) 283.

[11] G. Roelkens, D. Van Thourhout, R. Baets, R. Nötzel, and M. K. Smit: Opt. Express, 14, (2006) 8154.

[12] S. Stankovic, R. Jones, M. N. Sysak, J. M. Heck, G. Roelkens, and D. Van Thourhout: IEEE Photon. Technol. Lett., 23, (2011) 1781.

[13] N. Lindenmann, G. Balthasar, D. Hillerkuss, R. Schmogrow, M. Jordan, J. Leuthold, W. Freude, and C. Koos: Opt. Express, 20, (2012) 17667.

[14] C. Koos, J. Leuthold, W. Freude, N. Lindenmann, S. Koeber, G. Balthasar, J. Hoffmann, T. Hoose, P. Huebner, D. Hillerkuss, and R. Schmogrow: Proc. SPIE, 8613, (2013) 86130W.

[15] C. Koos, J. Leuthold, W. Freude, N. Lindenmann, S. Koeber, J. Hoffmann, T. Hoose, and P. Huebner: Proc. 15th International Conference on Transparent Optical Networks, (2013) 1202.

[16] S. Maruo, O. Nakamura, and S. Kawata: Opt. Lett., 22, (1997) 132.

[17] H. B. Sun, T. Kawakami, Y. Xu, J. Y. Ye, S. Matuso, H. Misawa, M. Miwa, and R. Kaneko: Opt. Lett., 25, (2000) 1110.

[18] M. Miwa, S. Juodkazis, T. Kawakami, S. Matsuo, and H. Misawa: Appl. Phys. A, 73, (2001) 561.

[19] X. M. Duan, H. B. Sun, K. Kaneko, and S. Kawata: Thin Solid Films, 453, (2004) 518.

[20] T. Amemiya, A. Ishikawa, Y. Shoji, P.N. Hai, M. Tanaka, T. Mizumoto, T. Tanaka, and S. Arai: Opt. Lett., 39,(2014) 212.

[21] A. Ishikawa and T. Tanaka: J. Laser Micro/Nanoeng., 7, (2012) 11.

[22] T. Okamoto, N. Nunoya, Y. Onodera, T. Yamazaki, S. Tamura, and S. Arai: IEEE J. Sel. Top. Quantum Electron. 9 (2003) 1361.

[23] N. Nunoya, M. Nakamura, M. Morshed, S. Tamura, and S. Arai: IEEE J. Sel. Top. Quantum Electron. 9 (2001) 249
[24] T. Shindo, M. Futami, K. Doi, T. Amemiya, N. Nishiyama, and S. Arai: IEEE J. Sel. Top. Quantum Electron., 19, (2013) 1502009.

[25] T. Shindo, M. Futami, T. Okumura, R. Osabe, T. Koguchi, T. Amemiya, N. Nishiyama, and S. Arai: IEEE Photon. Technol. Lett., 25, (2013) 1282.

[26] K. Doi, T. Shindo, J. Lee, T. Amemiya, N. Nishiyama, and S. Arai: IEEE J. Quantum Electron., 50, (2014) 321.

[27] T. Okumura, M. Kurokawa, M. Shirao, D. Kondo, H. Ito, N. Nishiyama, and S. Arai: Opt. Express, 17, (2009) 12564.

[28] T. Okumura, M. Kurokawa, H. Ito, D. Kondo, N. Nishiyama, and S. Arai: Proc. 21st International conference on Indium Phosphide and Related Materials, (2009) 178.

[29] N.D. Lai, J.H. Lin, P.W. Chen, J.L. Tang, C.C. Hsu: Opt. Comm., 258, (2005) 97.

(Received: June 17, 2014, Accepted: February 19, 2015) 